\title{
Combination of Vacuum and Solution Processes for Stable Indoor Organic Solar Cell under LED Illumination
}

\author{
Received 8 October, 2021; revised 9 November, 2021; accepted 10 November, 2021
}

\author{
Yongju Lee $^{a, b}$ (D), Swarup Biswas ${ }^{a}$ (D), and Hyeok Kim ${ }^{a, *(D)}$ \\ aschool of Electrical and Computer Engineering, Institute of Information Technology, University of Seoul, Seoul O2504, \\ Republic of Korea \\ bApplied Robot R\&D Department, Korea Institute of Industrial Technology (KITECH), Ansan 15588, Republic of Korea
}

\author{
*Corresponding author E-mail: hyeok.kim@uos.ac.kr
}

\begin{abstract}
The use of tiny, micro-powered electronic devices in indoor environments is increasing daily. The demand for high-performing organic photovoltaics (OPVs) to power these devices has been rapidly growing because OPVs have favorable absorption properties for indoor environments, excellent mechanical flexibility, and other advantageous attributes. Recently, inexpensive, less acidic, and water-processable polyaniline:poly (4-styrenesulfonic acid) (PANI:PSS) was shown to deliver remarkably high performance as the hole transport layer (HTL) in an indoor OPV device with poly(3-hexylthiophene): indene-C60 bisadduct as the active layer. However, PANI:PSS has not been tested in combination with other active materials, although its universal applicability is of key importance for its commercialization. Thus, we tested the potentiality of using PANI:PSS as the HTL of a highly suitable active material, poly[[4,8-bis[(2-ethylhexyl)oxy]benzo[1,2-b:4,5-b']dithiophene-2,6-diyl][3-fluoro-2[(2-ethylhexyl-1)carbonyl]thieno[3,4-b]thiophendiyl]:phenyl-C70-butyric acid methyl ester (PTB7:PC 70 BM) in an OPV device under an LED lamp. The results reveal that the OPV device based on the PANI:PSS HTL and PTB7:PC ${ }_{70} \mathrm{BM}$ as the active material has high efficiency (11.80\%) and a maximum power density of $42.5 \mu \mathrm{W} / \mathrm{cm}^{2}$ under a 1,000 lx LED.
\end{abstract}

Keywords: Indoor organic photovoltaic cell, Vacuum and solution process, LED lamp, Hole transport layer, Polyaniline

\section{Introduction}

The demand for Internet of Things (IoT) networks is rapidly increasing $[1,2]$. The constituent devices of an IoT network are small, low-powered ( 20 to $50 \mu \mathrm{W}$, on average), and wireless. Among the different energy-harvesting technologies, the use of photovoltaic (PV) devices to harvest indoor light energy is one of the best options for powering these devices [3-5].

Lamps based on different illuminating agents, such as light-emitting diodes (LEDs) and halogen, incandescent, and fluorescent materials, are widely used to light indoor environments $[6,7]$. These light sources have an intensity of approximately 0.01 times that of a one-sun light source, and the wavelength bands are different for each of these types of light sources (Fig. 1).

Organic photovoltaics (OPVs) are good candidates to power IoT devices owing to their excellent spectral matching (between the incident and absorption spectra), as well as their ultrathin and flexible nature [8-10]. Although significant research has been conducted to advance indoor OPVs, these devices have not yet been commercialized owing to their shorter lifetimes, instability, and lack of universality (i.e., being equally efficient for various light sources).

In an OPV device, the hole transport layer (HTL) plays a crucial role during operation [11], preventing charge carrier recombination and enhancing the movement of holes toward the anode [11]. Most researchers have used poly (3, 4-ethylenedioxythiophene) (PEDOT:PSS) doped with polystyrene sulfonate as the HTL of OPVs owing to its excellent heat resistance, good electrical properties, and very good

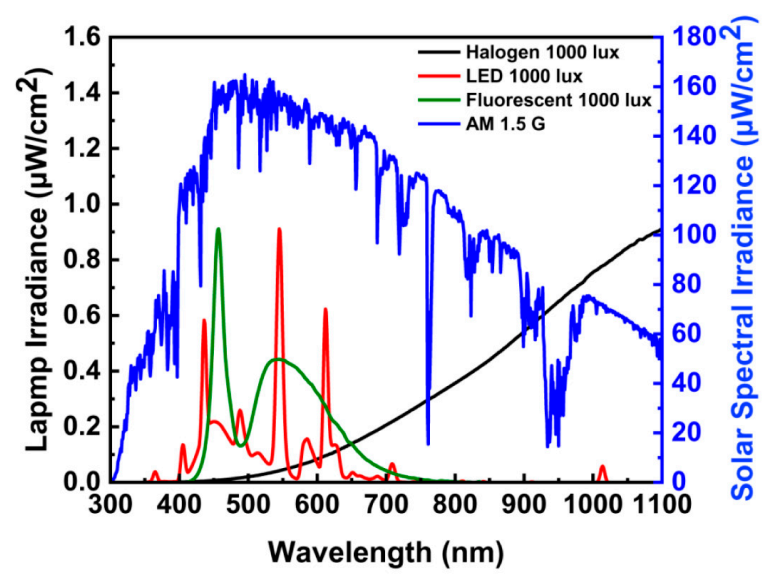

Figure 1. Emission spectra of different outdoor and indoor light sources.

transmittance in the visible range [12]. However, the utilization of PEDOT:PSS as HTL negatively affects the device performance (faster increase in its series resistance (Rs), degradation, etc.), as it is highly acidic ( $\mathrm{pH}=1.6)$ and hygroscopic [13]. Furthermore, the fact that it is expensive to purchase increases the device fabrication cost.

Considering these issues, Biswas et al. developed a low-cost, less acidic $(\mathrm{pH}=2.2)$, and easy-to-process organic polymeric semiconduc- 


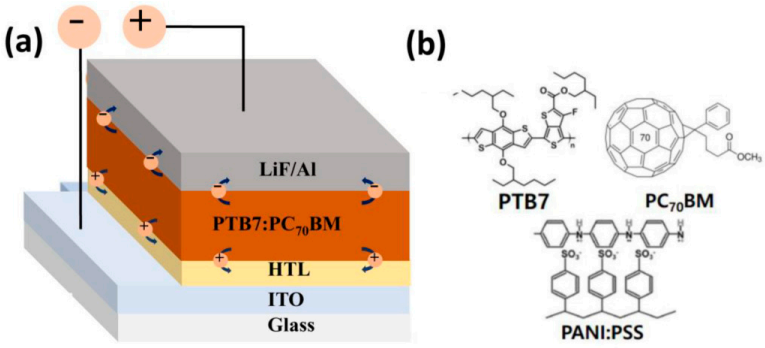

Figure 2. (a) Device structure of PTB7:PC 70 BM based OPV. (b) Chemical structure of PTB7 and PC 70 BM.

tor, polyaniline doped with poly(4-styrenesulfonic acid) (PANI:PSS), and demonstrated that it could be used as the HTL of an OPV with poly(3-hexylthiophene): indene-C60 bisadduct (P3HT:ICBA) as the active layer. The power conversion efficiency (PCE) of the device under an LED lamp was remarkably high [14-16]. Moreover, the device had a longer lifetime than the commercially available HTL, i.e., an OPV based on PEDOT:PSS [15]. Apart from its highly promising properties, its universality (i.e., compatibility with other active materials) has not yet been tested, although a commercial device with these properties is in great demand [15].

Among the various donor materials, poly[[4,8-bis[(2-ethylhexyl) oxy]benzo[1,2-b:4,5-b'] dithiophene-2,6-diyl][3-fluoro-2-[(2-ethylhexyl)carbonyl]thieno[3,4-b]thiophenediyl] (PTB7) is one of the best organic semiconductors (polymer) for use in indoor OPVs owing to its wide absorption band (400 to $750 \mathrm{~nm}$ ) and superior photostability [17]. The optical energy band gap of phenyl-C70-butyric acid methyl ester $\left(\mathrm{PC}_{70} \mathrm{BM}\right)$ corresponds excellently with that of PTB7 (which is necessary for constructing a well-behaved PV device) [17]. Moreover, LED lamps are widely used to illuminate offices, malls, bedrooms, living rooms, and other spaces because of their lower power consumption, longer lifetime, and cost effectiveness. Thus, highly efficient and stable OPVs that are compatible with LED lights would have to be developed [18].

This motivated us to test the feasibility of using PANI:PSS as the HTL of an OPV based on PTB7:PC ${ }_{70} \mathrm{BM}$ as the active material to harvest the indoor light generated by an LED lamp. Towards this goal, we synthesized water-stable PANI:PSS and fabricated OPVs with PTB7:PC ${ }_{70} \mathrm{BM}$ as the active material and PANI:PSS as the HTL. Then, we tested the solar cells under a 1,000 lx LED. Our results showed that OPV devices with PANI:PSS as the HTL and PTB7:PC ${ }_{70} \mathrm{BM}$ as the active material exhibit very high PCE $(11.80 \%)$ and a maximum power density of $42.5 \mu \mathrm{W} / \mathrm{cm}^{2}$ under a 1,000 lx LED lamp.

\section{Experimental details}

\subsection{Synthesis of HTL material}

Here, we prepared PANI:PSS via a simple chemical route we reported earlier [16]. First, a mixture consisting of aniline (from Sigma Aldrich, St. Louis, USA) and water was prepared by adding doubledistilled aniline $[2.15 \mathrm{mM}(0.20 \mathrm{~g})]$ to $50 \mathrm{~mL}$ of deionized (DI) water and keeping the mixture at $0-5{ }^{\circ} \mathrm{C}$ for $1 \mathrm{~h}$ while stirring $(1,000 \mathrm{rpm})$. Afterward, $0.20 \mathrm{~g}$ of PSS was added to this solution and the resultant mixture was stirred $(1,000 \mathrm{rpm})$ for $5 \mathrm{~h}$ while maintaining the temperature constant at $0-5^{\circ} \mathrm{C}$. Simultaneously, a solution of ammonium persulfate (APS) (from Sigma Aldrich) was prepared by dissolving $2.15 \mathrm{mM}$ of APS into $25 \mathrm{~mL}$ of DI water by vigorously stirring for $30 \mathrm{~min}(1,000 \mathrm{rpm})$. Then, we preserved the APS solution in a refrigerator $\left(0-5{ }^{\circ} \mathrm{C}\right)$ for $5 \mathrm{~h}$. Subsequently, the aniline was attached to the PSS (as a result of the attracting force between the ammonium $\left(\mathrm{NH}_{4}{ }^{+}\right)$group of aniline and the sulfite $\left(\mathrm{SO}_{3}{ }^{-}\right)$group of PSS) while (a)

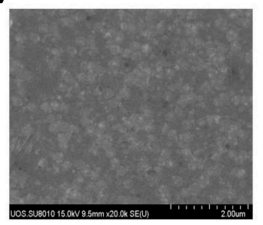

(b)

(c)

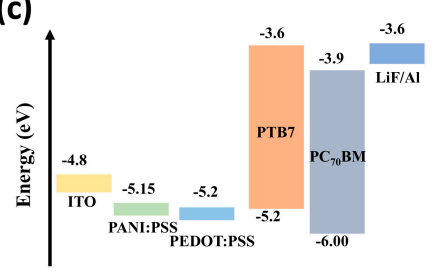

Figure 3. (a) SEM image of PANI:PSS. (b) AFM image of PANI:PSS. (c) Energy-level diagrams of OPV components.

vigorously stirring $(1,000 \mathrm{rpm})$ the mixture for $24 \mathrm{~h}$ at $0-5{ }^{\circ} \mathrm{C}$ to complete the polymerization. Lastly, the PANI:PSS was formed as a dark green stable solution. The sample was stored in the refrigerator for further utilization. We chose the weight ratio of PANI to PSS as 1:1 because it was observed that PANI:PSS with this particular weight ratio had the highest conductivity and best performance level as the HTL of an OPV [16].

\subsection{Preparation of solution}

A solution containing $25 \mathrm{mg}$ PTB7:PC ${ }_{70} \mathrm{BM}(1: 1.5 \mathrm{w} / \mathrm{w} ; 10 \mathrm{mg}$ of PTB7 and $15 \mathrm{mg}$ of $\mathrm{PC}_{70} \mathrm{BM}$ ) in $1 \mathrm{~mL}$ of chlorobenzene (from Sigma Aldrich) was prepared by stirring at $50{ }^{\circ} \mathrm{C}$ for $12 \mathrm{~h}$ under a nitrogen atmosphere in a glove box (GB). Both the donor and acceptor were collected from the same material.

\subsection{Device fabrication}

We fabricated the OPV device [Fig. 2(a)] by combining the techniques of vacuum and solution processing. Initially, an ITO-coated glass substrate was cleaned by ultra-sonication in a detergent bath and rinsing with DI water. Again the substrate was cleaned successively using ultra-sonication in a bath containing different solvents (DI water, acetone, and 2-propanol). After that we dried the substrate in a stream of nitrogen gas $\left(\mathrm{N}_{2}\right)$. Next, we exposed the substrate to an $\mathrm{O}_{2}$ plasma $(900 \mathrm{~s})$, and then deposited the HTL of the device by spin coating $(4,000 \mathrm{rpm}$ for $60 \mathrm{~s})$ PANI:PSS (filtered through a PVDF syringe filter $(0.45 \mu \mathrm{m}))$ onto the substrate. The substrate was heated for $30 \mathrm{~min}$ at $130^{\circ} \mathrm{C}$ before it was transferred to a $\mathrm{GB}\left(\mathrm{N}_{2}\right.$ filled). The PTB7:PC ${ }_{70} \mathrm{BM}$ solution (filtered through a PTFE filter $\left.(0.45 \mu \mathrm{m})\right)$ was then spin-coated onto the substrate at 3,000 $\mathrm{rpm}$ for $30 \mathrm{~s}$. The substrate was again heated for $30 \mathrm{~min}$ in the $\mathrm{GB}\left(130^{\circ} \mathrm{C}\right)$. Then, a shadow mask was used to form a lithium fluoride $(\mathrm{LiF})$ layer $(0.5 \mathrm{~nm}$ thick) on the PTB7:PC ${ }_{70} \mathrm{BM}$ layer using vacuum thermal evaporation (the evaporation system was attached to the GB). The deposition rate and base pressure was $0.01-0.02 \mathrm{~nm} / \mathrm{s}$ and $1 \mu \mathrm{Pa}$, respectively. Subsequently, we deposited a $100 \mathrm{~nm}$ thick $\mathrm{Al}$ layer onto the $\mathrm{LiF}$ layer using vacuum thermal deposition (at a rate of 0.5 to $0.6 \mathrm{~nm} / \mathrm{s}$ and pressure of $1 \mu \mathrm{Pa}$ ). The active area of the PV device was $0.0225 \mathrm{~cm}^{2}$. The chemical structures of the donor, acceptor, and HTL material are illustrated in Fig. 2(b). For comparison, we also fabricated a PEDOT:PSS (Ossila, Sheffield, UK) HTL-based OPV device using the same procedure. 


\subsection{Characterization of the material and device}

High-resolution scanning electron microscopy (SEM) images of the HTL were recorded by field-emission SEM (JEOL, JSM 7610F). Surface profiles of the PANI:PSS film were acquired by using atomic force microscopy (AFM, PSIA XE-100). An optical microscope was used to measure the active area of the devices. Current density (J) to voltage $(\mathrm{V})$ characteristic curves of the OPVs (under 1,000 lx LED lamps) were recorded using a Keithley 2401 source meter. A solar simulator (McScience, Suwon, Republic of Korea) was used as the light source, with a 1,000 lx LED lamp (white linear COB LED from McScience) with irradiance of $280 \mu \mathrm{W} / \mathrm{cm}^{2}$.

\section{Results and discussion}

The HTL (i.e., PANI:PSS), of which the concentration of PSS had been optimized, was synthesized via a simple chemical route we reported previously. Therefore, we did not fully characterize the material this time [16]. After synthesizing the PANI:PSS, we deposited the material onto the cleaned glass substrates to form a film and recorded the SEM and AFM images of the film [Figs. 3(a) and 3(b), respectively]. As shown in the figures, the surface of the PANI:PSS film is slightly irregular. The rms value of the surface roughness of the film is 35.4 $\mathrm{nm}$, which is quite similar to that in the previous report [16]. On the other hand, the commercially available HTL based on PEDOT:PSS has a surface roughness value of approximately $1 \mathrm{~nm}$ [16]. This is one disadvantage of using PANI:PSS as the HTL. We expect a further decrease in the surface roughness of the HTL based on PANI:PSS to increase the PCE of the corresponding OPV.

Figure 3(c) depicts the energy band alignment of the different layers of the OPV. The values of the energy levels of the different constituent materials were obtained from previous reports $[19,20]$. The highest occupied molecular orbital (HOMO) level of PTB7 is very close to that for PANI:PSS, which is highly desirable for transporting holes toward the anode. Furthermore, the good correspondence between the lowest unoccupied molecular orbital (LUMO) of $\mathrm{PC}_{70} \mathrm{BM}$ and the work function of $\mathrm{LiF}$ is favorable for the movement of electrons toward the cathode.

As we were more interested in the performance of PV devices that were exposed to LED light, we subjected the devices to additional tests under this illuminating condition. The corresponding J-V curves for devices with different HTLs (PANI:PSS, PEDOT:PSS) (one representative curve) are presented in Fig. 4(a). The corresponding device performance parameters and standard deviation of the error value (six devices) are provided in Table I. The PCE values of the OPV cells were estimated using Eq. (1):

$$
P C E=\frac{M P D}{P_{\text {in }}} \times 100=\frac{V_{O C} \times J_{S C} \times F F}{P_{\text {in }}}
$$

where $M P D$ is the maximum power generated by the device, $P_{i n}$ is the irradiance power intensity of the incident light, $V_{O C}$ is the opencircuit voltage, $J_{S C}$ is the short-circuit current density, and $F F$ is the fill factor.

Additionally, Fig. 4(b) visualizes the deviation in device performance (difference between the best and worst performing device) when changing the position of the device on the substrate for the devices of

Table I. Summary of different OPV device performance (operating under the 280 $\mu \mathrm{W} / \mathrm{cm}^{2}(1,000 \mathrm{~lx})$ LED lamp.) parameters.

\begin{tabular}{ccccccc}
\hline HTM & $\begin{array}{c}\text { Voc } \\
(\mathrm{mV})\end{array}$ & $\begin{array}{c}\text { Jsc } \\
\left(\mu \mathrm{A} / \mathrm{cm}^{2}\right)\end{array}$ & $\begin{array}{c}\text { FF } \\
(\%)\end{array}$ & $\begin{array}{c}\text { PCE } \\
(\%)\end{array}$ & $\begin{array}{c}\mathrm{MPD} \\
\left(\mu \mathrm{W} / \mathrm{cm}^{2}\right)\end{array}$ & $\begin{array}{c}\mathrm{R}_{S} \times \mathrm{A} \\
\left(\Omega \times \mathrm{cm}^{2}\right)\end{array}$ \\
\hline $\begin{array}{c}\text { PANI: } \\
\text { PSS }\end{array}$ & $612 \pm 3.5$ & $98.4 \pm 1.2$ & $61.3 \pm 1.2$ & $11.5 \pm 0.3$ & 42.5 & 41377 \\
$\begin{array}{c}\text { PEDOT: } \\
\text { PSS }\end{array}$ & $640 \pm 2.4$ & $109.2 \pm 8.0$ & $65.1 \pm 0.4$ & $14.2 \pm 1.1$ & 50.0 & 34932
\end{tabular}

(a)

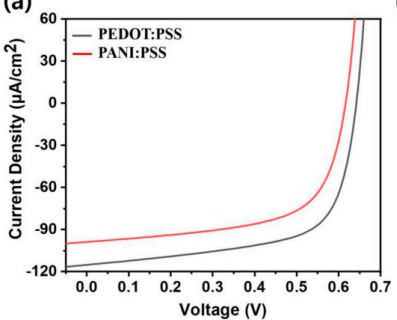

(b)

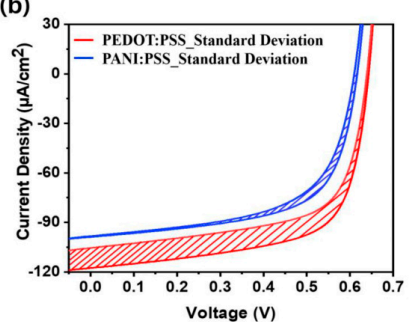

Figure 4. (a) Representative J-V characteristics of best performing OPVs (based on PEDOT:PSS and PANI:PSS HTL) evaluated under the illumination of a 1,000 lX LED lamp. (b) Comparison of the deviation (J-V curve of the best- and worstperforming devices) in the performance of both PEDOT:PSS and PANI:PSS HTLbased OPVs.

which the HTL is based on both PANI:PSS and PEDOT:PSS. Here it is notable that the performance level for all six devices (based on the PANI:PSS HTL) is nearly the same, implying that the uniformity in the physiochemical properties of the PANI:PSS film is excellent and for PEDOT:PSS this deviation is higher. This implies a greater nonuniformity in the physiochemical properties of PEDOT:PSS compared with PANI:PSS. As presented in Table I, the highest PCE value of the OPV with the HTL based on PANI:PSS is $11.80 \%(11.50 \pm 0.30 \%)$. The corresponding $M P D, V_{O C}, J_{S C}$, and $F F$ values are $42.50 \mu \mathrm{W} / \mathrm{cm}^{2}$, $612 \pm 3.53 \mathrm{mV}, 98.40 \pm 1.18 \mu \mathrm{A} / \mathrm{cm}^{2}$, and $61.27 \pm 1.22 \%$, respectively. Table I also indicates that the PEDOT:PSS-based reference OPV can exhibit a $14.23 \pm 1.11 \%$ PCE value with $50 \mu \mathrm{W} / \mathrm{cm}^{2}, 640 \pm 2.37 \mathrm{mV}$, $109.2 \pm 8.04 \mu \mathrm{A} / \mathrm{cm}^{2}$, and $65.13 \pm 0.43 \%$ for the $M P D, V_{O C}, J_{S C}$, and $F F$ values, respectively. Previously, the OPVs based on PANI:PSS exhibited higher environmental stability than the PEDOT:PSS-based OPVs (P3HT:ICBA active layer) owing to their lower acidity $(\mathrm{pH}=2.2)$ and because they are less hygroscopic. Therefore, the OPVs can be expected to have a longer lifetime than the device of which the HTL is based on PEDOT:PSS. Moreover, the production cost of PANI:PSS is significantly lower than that of the PEDOT:PSS [16]. Although the PCE of the OPV based on PANI:PSS is slightly lower than that of the PEDOT:PSS-based device, PANI:PSS is a highly promising HTL for an OPV in terms of its longer lifetime and lower production cost. Table I indicates that the OPV with the PANI:PSS HTL has a significantly high $M P D\left(42.50 \mu \mathrm{W} / \mathrm{cm}^{2}\right)$ value, sufficient for operating different electronic devices (micropowered) attached to an IoT network.

\section{Conclusions}

We investigated the potential of using PANI:PSS as the HTL of an OPV with PTB7:PC ${ }_{70} \mathrm{BM}$ as the active material for operation under an LED lamp. First, we successfully synthesized the PANI:PSS via a simple chemical route. Then, we fabricated OPVs with PTB7:PC 70 BM as the active material and PANI:PSS as the HTL using a combination of vacuum and solution processes. The analysis of the test results of the OPV (for operation under a 1,000 lx LED lamp) revealed that the OPV with the PANI:PSS-based HTL had very high PCE $(11.80 \%)$ and maximum power density $\left(42.50 \mu \mathrm{W} / \mathrm{cm}^{2}\right)$.

\section{Acknowledgments}

This research was supported by the International Research \& Development Program of the National Research Foundation of Korea (NRF) funded by the Ministry of Education, Science and Technology(MEST) of Korea (2019K1A3A1A21031246). Furthermore the Korea Institute of Industrial Technology under the "Development of Soft Robotics Technology for Human-Robot Coexistence Care Robots" (Grant Number: KITECH EH210010) also funded the research work 


\section{Conflicts of Interest}

The authors declare no conflicts of interest.

\section{ORCID}

Yongju Lee

Swarup Biswas

Hyeok Kim https://orcid.org/0000-0001-9128-2450 https://orcid.org/0000-0003-1120-1433 https://orcid.org/0000-0003-2164-2849

\section{References}

[1] M. H. Kashani, M. Madanipour, M. Nikravan, P. Asghari, and E. Mahdipour, J. Netw. Comput. Appl. 192, 103164 (2021)

[2] H. Landaluce, L. Arjona, A. Perallos, F. Falcone, I. Angulo, and F. Muralter, Sensors 20, 2495 (2020).

[3] H. K. Lee, Z. Li, J. R. Durrant, and W. C. Tsoi, Appl. Phys. Lett. 108, 253301 (2016).

[4] S. S. Yang, Z. C. Hsieh, M. L. Keshtov, G. D. Sharma, and F. C. Chen, Sol. RRL 1, 1700174 (2017).

[5] H. Elahi, K. Munir, M. Eugeni, S. Atek, and P. Gaudenzi, Energies 13, 5528 (2020)

[6] K. Yoo, S. Biswas, Y. J. Lee, S. C. Shin, K. J. Kim, J. W. Shim, H. Kim, and J. J. Lee, IEEE Access 8, 114752 (2020).

[7] X. Ma, S. Bader, and B. Oelmann, IEEE Sens. J. 17, 3884 (2017).

[8] J. Hou et al., Mater. Chem. Front. 5, 893 (2021).
[9] Y. Cui, H. Yao, T. Zhang, L. Hong, B. Gao, K. Xian, J. Qin, and J. Hou, Adv. Mater. 31, 1904512 (2019).

[10] S. Biswas and H. Kim, Polymers 12, 1338 (2020).

[11] M. T. Lloyd, C. H. Peters, A. Garcia, I. V. Kauvar, J. J Berry, M. O. Reese, M. D. McGehee, D. S. Ginley, and D. C. Olson, Sol. Energy Mater. Sol. Cells 95, 1382 (2011).

[12] M. Ritruksa, S. Wongrerkdee, K. Lohawet, A. Kaewprjak, P. Kumnorkaew, and S. Wongrerkdee, Surf. Interfaces 21, 100716 (2020).

[13] O. Abdulrazzaq, S. E. Bourdo, M. Woo, V. Saini, B. C. Berry, A. Ghosh, and A. S. Biris, ACS Appl. Mater. Interfaces 7, 27667 (2015).

[14] S. Biswas, Y. J. You, Y. J. Lee, J. W. Shim, and H. Kim, Dyes. Pigm. 183,108719 (2020).

[15] S. Biswas, Y. J. You, J. W. Shim, and H. Kim, Thin Solid Films 700, $137921(2020)$

[16] S. Biswas, Y. J. You, J. S. Kim, S. R. Ha, H. S. Choi, S. H. Kwon, K. K. Kim, J. W. Shim, and H. Kim, Appl. Surf. Sci. 512, 145700 (2020).

[17] Y. J. You, C. E. Song, Q. V. Hoang, Y. M. Kang, J. S. Goo, D. H. Ko, J. J. Lee, W. S. Shin, and J. W. Shim, Adv. Funct. Mater. 29, 1901171 (2019).

[18] H. S. Ryu et al., J. Mater. Chem. A 8, 23894 (2020).

[19] T. A. Shastry, I. Balla, H. Bergeron, S. H. Amsterdam. T. J. Marks, and M. C. Hersam, ACS Nano 10, 10573 (2016).

[20] S. Mori, T. Gotanda, Y. Nakano, M. Saito, K. Todori, and M. Hosoya, Jpn. J. Appl. Phys. 54, 071602 (2015). 